\title{
L'Inferno nel ciclo dei Contorni danteschi di Bonaventura Genelli
}

\author{
Antonella Ippolito \\ Universität Potsdam \\ ippolito@uni-potsdam.de
}

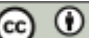

\section{Riassunto}

Il ciclo dei trentasei Contorni danteschi del pittore e disegnatore berlinese Giovanni Bonaventura Genelli (I798-I868) rappresenta un interessante tentativo d'interpretare il testo della Commedia alla luce dei principi del classicismo tedesco, sull'esempio di Carstens e di Koch e, in parte di Flaxman da cui però Genelli si distingue per continua ricerca volta ad esprimere la plasticità delle forme. Caratteristica precipua delle sue illustrazioni è inoltre il ricorso continuo sia alla scultura antica che agli affreschi michelangioleschi della Sistina come repertorio iconografico. Larticolo approfondisce questi aspetti con particolare riferimento alle sedici tavole dedicate all'Inferno.

Parole chiave: Inferno, illustrazione, disegno, Neoclassicismo, arte tedesca.

\begin{abstract}
The cycle of the 36 Outline illustration to Dante's Divine Comedy represents a very interesting attempt towards a pictorial interpretation of the text inspired to the principles of the German classicism. Genelli is in this respect a follower of Carstens and Koch. The cleanliness of his line drawing with no shading and few background details is reminiscent of Flaxman; nonetheless, an evident mark of Genelli's style is his permanent effort of exploring and expressing the dynamic plasticity of bodies. To represent Dante's world he also draws on ancient sculpture as well as on Michelangelo's Sistina frescoes as an iconographic repertoire. These aspects are here illustrated with special regard to the sixteen illustration of the Inferno.
\end{abstract}

Key words: Inferno, Illustration, Drawing, Neoclassicism, German art. 
Der la sua Dante-Symphonie, concepita fin dal I837 e composta tra il I855 e il 1857 , Franz Liszt aveva in mente un impianto multimediale ante litteram, destinato ad evidenziare i contenuti poetico-drammatici del testo attraverso una sintesi di musica, testo e immagine. Al centro di questo progetto stava la realizzazione di un Diorama. La proiezione di immagini su uno schermo trasparente, che simulava attraverso giochi di luce il movimento e le variazioni di luogo e di tempo, godeva di crescente popolarità come mezzo per ottenere effetti di realismo e creare particolari atmosfere nella messa in scena di opere e pièces teatrali. Liszt si proponeva di utilizzare questo sistema per far scorrere davanti agli occhi del pubblico, contemporaneamente all'esecuzione della sinfonia, le scene centrali del viaggio ultraterreno di Dante.

L'ambizioso progetto occupò il compositore almeno dal i847. Tuttavia, esso non si spinse oltre i primi abbozzi: furono soprattutto i costi esorbitanti a dissuadere Liszt dal perseguirlo oltre, soprattutto dopo che la contessa Caroline von Sayn-Wittgenstein, che in un primo momento aveva entusiasticamente appoggiato l'idea, fu costretta da difficoltà economiche a ritirare il proprio sostegno. Ne sopravvive però una traccia sulla copertina di un'edizione del 1920 della Sinfonia "Dante", su cui appare un disegno dell'artista che avrebbe dovuto realizzarlo.

\section{II}

La scelta, come apprendiamo dalle lettere di Liszt, era caduta sul berlinese Giovanni Bonaventura Genelli. La concezione artistica di questo pittore, e disegnatore, noto per le sue composizioni cicliche ad acquarello, a penna, a grafite e ad incisione ${ }^{\mathrm{I}}$, doveva apparire a Liszt particolarmente adeguata alla realizzazione del progetto, per il fatto che presentava molti punti di contatto con la sua idea di musica come linguaggio poetico-pittorico. Per la sua opera, concepita secondo canoni formali classicistici, Genelli privilegiava la sinteticità lineare del semplice contorno. La rinuncia ad una netta distinzione tra luci e ombre, ai colori ed al disegno interno consentiva una rappresentazione estremamente ridotta e solo limitatamente mimetica, atta a stimolare l'immaginazione dell'osservatore. Le sue creazioni si basavano inoltre in prevalenza sul principio della narrazione ciclica: in essa Genelli riconosceva la forma di rappresentazione che nel modo più chiaro rifletteva la "disposizione poetica" quale elemento fondamentale del lavoro artistico. In quello che Peter

I. Di questa produzione fanno parte, oltre alle illustrazioni dei poemi omerici (realizzate tra il I837 e il I849), i tre "cicli poetici" Aus dem Leben eines Wüstlings (Scene dalla vita di un dissoluto, 1840), Aus dem Leben einer Hexe (Scene dalla vita di una strega, 1843), Aus dem Leben eines Künstlers (Scene dalla vita di un artista, completato nel I86I). 
Cornelius $^{2}$ definiva come il "bello e nobile lavoro di rielaborazione creativa a partire da materiali poetici già completa" ("schöne und edle Arbeit des Wiederdichtens aus vollendeten Poesie") egli vedeva, in accordo con i postulati winckelmanniani dell'imitazione ${ }^{3}$, la "parte più essenziale dell'arte" ("das Wesentlichste in der Kunst"4). Questo spiega la sua predilezione per la trasposizione pittorica delle grandi opere della letteratura mondiale, che già aveva dato luogo, oltre a un gran numero di composizioni di argomento biblico, a due cicli di disegni sui poemi omerici. Nel novero dei temi di Genelli rientrava anche l'opera di Dante. Contemporaneamente all'elaborazione del progetto di Liszt, egli aveva prodotto anche un ciclo dedicato alla Divina Commedia, che con grande probabilità il compositore aveva avuto occasione di vederes.

\section{III}

Nato a Berlino nel 1798 da una famiglia di artisti, i cui antenati si erano trasferiti dall'Italia inizialmente in Danimarca e in un secondo momento in Germania, Giovanni Bonaventura Genelli nutriva per Dante un interesse che risaliva alla prima giovinezza ed era in primo luogo dovuto all'influsso dello zio paterno, il celebre architetto e scrittore Hans-Christian Genelli. Questi lo aveva non solo introdotto alla lettura del poeta italiano, ma gli aveva anche fatto conoscere i disegni danteschi di Asmus Jakob Carstens, con cui era stato legato da amicizia. Un'importanza decisiva ebbe tuttavia il soggiorno decennale del giovane artista (I822-I832) in Italia. Giunto a Roma per approfondire lo studio dell'arte antica e rinascimentale, egli trovò un paterno amico e mentore in Joseph Anton Koch, che in quello stesso periodo stava lavorando agli affreschi a tema dantesco del Casino di Villa Massimo a Roma, e rimase fortemente influenzato dalla sua opera.

Al rientro dall'Italia, Genelli si stabilì a Monaco, dove a dispetto di notevoli difficoltà finanziarie produsse numerosi disegni ed illustrazioni. In questo periodo, ebbe modo di ascoltare nel capoluogo bavarese alcune conferenze pubbliche di Rudolf Markgraff sulla Divina Commedia, che gli ispirarono diverse composizioni dantesche tra loro indipendenti. Quando queste divennero note tra gli artisti di Monaco, Genelli venne invitato ad illustrare il capolavo-

2. Cornelius (I869: 803; trad. AI).

3. Cf. J.J. Winckelmann, Gedanken über die Nachahmung der griechischen Werke in der Malerei und Bildhauerkunst (I755).

4. Lettera di Genelli a Karl Friedrich Schinkel, 30.12.1828.

5. Genelli era in contatto epistolare con Liszt. Una lettera del 1853 , in cui egli risponde negativamente alla richiesta del compositore d'ingrandire un disegno (non precisato) potrebbe essere in rapporto con il progetto del Diorama. A questo proposito si veda Mungen (2006: 309, nota I23). 
ro di Dante, come già aveva fatto con l'Iliade e l'Odissea; una sottoscrizione collettiva avrebbe garantito la copertura dei costi ${ }^{6}$.

Tra il I846 e il I849 vide così la luce un corpus di 36 disegni, inizialmente diffusi in singole edizioni di 4 tavole per volta, e solo in un secondo momento pubblicati come ciclo completo. La raccolta fu poi edita nel I849 con il titolo Contorni sulla Divina Commedia di Dante (Umrisse zu Dantes Göttlicher Commödie), con dedica al principe Johann di Sassonia, che poco tempo prima, con lo pseudonimo di Filalete, aveva pubblicato la sua nota traduzione in tedesco del testo di Dante. A questa edizione, per molti versi deludente per l'artista ${ }^{7}$, ne seguì sei anni dopo una seconda con prefazione di Max Jordan, apparsa in occasione del seicentesimo anniversario della nascita del poeta e meglio recepita da parte del pubblico: a ciò contribuì probabilmente la presenza in questo volume dei versi di Dante di volta in volta illustrati e di semplici commenti esplicativi in tedesco e in francese.

I disegni originali di Genelli, eseguiti ad inchiostro su carta gialla, bianca e bruna, sono oggi conservati nel Gabinetto delle Stampe di Dresda. Altre 24 tavole, quasi identiche, si trovano inoltre nella Collezione Grafica della Klassik-Stiftung di Weimar, dove l'artista trascorse gli ultimi dieci anni della sua vita al servizio dell'arciduca Carl Alexander di Sassonia-Weimar ${ }^{8}$. Questi meno noti esemplari sono ritenuti per la maggioranza copie posteriori, anche se la questione del rapporto con i disegni di Dresda andrebbe ulteriormente indagata.

Dell'insieme delle tavole, I6 si riferiscono all'Inferno, i2 al Purgatorio e 8 al Paradiso. L'interesse prevalente per la prima cantica, che Genelli condivide con Koch, si spiega solo in parte con la difficoltà di trasporre in immagini i temi sempre più astratti del Purgatorio e del Paradiso: era in particolare il ruolo preponderante dell'elemento umano, come pure il vasto repertorio di motivi mitologici, a rendere l'Inferno un soggetto particolarmente interessante

6. Lettera collettiva a firma di M. Widemann, G. Jäger, G. König, H. Merz, L. Lange, L. Schaller, in Ebert (1971: 93).

7. In una lettera del I6 novembre I847 a Giovanni Morelli, l'artista lamenta la qualità delle incisioni realizzate a partire dai suoi disegni, come pure quella del pubblico, poco favorevole ad una ricezione di Dante: "Dato che la maggior parte della gente non ha mai letto Dante, o se lo ha fatto, si è annoiata nella lettura, richiedere che essa debba provare un grande interesse di fronte alle raffigurazioni (del suo testo), specialmente se sono di buona qualità, è pretendere troppo" (Trad. AI).

8. Per l'arciduca Genelli produsse, accanto alla decorazione di un sipario teatrale (1866) alcuni dipinti a olio a tema biblico e mitologico (Visione d'Ezechiele, I857; Ratto di Europa, 1859; Ercole Musagete, I862; Abramo e l'angelo, I862; Bacco fugge da Licurgo, I864; Bacco tra le Muse, 1868), che restano una minoranza nell'ambito della sua vasta opera complessiva (circa I60o opere) composta soprattutto di disegni. 
per Genelli, nella cui intera opera artistica l'uomo e il mito rappresentano le due costanti decisive.

La selezione degli episodi` seguiva i criteri teorici già formulati da Johann Heinrich Meyer nel suo saggio Sugli oggetti dell'arte (Über die Gegenstände in den bildenden Künsten, I798). Fondamentale nell'ambito della rappresentazione ciclica era, secondo Meyer, una trattazione dei punti più significativi della storia da illustrare che tuttavia ne rappresentasse momenti non troppo distanti tra loro, così da consentire all'osservatore di non perdere il filo della narrazione. La riproduzione degli eventi narrati doveva inoltre conferire evidenza alle figure principali del testo. In accordo con questi principi, Genelli si concentrò quindi su figure ed episodi che presentavano un alto grado di drammaticità oppure erano caratterizzati da una forte presenza di figure favolose o mitologiche. Inoltre, egli scelse di illustrare parti del testo che permettessero una certa continuità narrativa tra le singole tavole, così che, come vedremo, due gruppi d'immagini si possono identificare nell'Inferno come "cicli nel ciclo". Per la stessa ragione, egli privilegia nel Purgatorio l'incontro di Dante con Buonconte da Montefeltro, al quale dedica due tavole, e nel Paradiso la storia di Francesco d'Assisi, estesa per un totale di cinque su otto disegni.

\section{V}

I. Anziché dal primo canto, il ciclo di disegni sull'Inferno prende avvio direttamente dal terzo, con l'immagine di Dante e Virgilio mentre attraversano l'Acheronte sulla barca con cui Caronte trasporta i dannati. La scena si presenta all'inizio piuttosto statica. Diversamente che nell'interpretazione di altri illustratori come Koch, Flaxman, Blake, i quali la inseriscono in uno spazio molto ampio e accentuano il senso del movimento, la barca non costituisce l'elemento dominante dell'immagine. Appena accennata, essa quasi scompare dietro alla massa dei corpi nudi, alcuni dei quali, rappresentati di spalle, sono utilizzati nella funzione di repoussoirs per accrescere il senso della profondità spaziale. Anche il motivo del vento, prevalente in Flaxman e Koch, è qui affidato unicamente al movimento dei capelli delle figure.

La composizione è determinata nel suo equilibrio dalla forma del suo supporto cartaceo. Genelli fa in modo che lo sguardo dell'osservatore, partendo dalle "anime [...] ch'eran lasse e nude" (If. III, IOo) arrivi gradualmente ad identificare Caronte, in piedi a destra, come la figura fondamentale dell'im-

9. Un po' superficialmente Crass (I981: 26): "I canti sono presi con un criterio arbitrario dall'intera opera, alcuni non vengono selezionati, ad altri invece toccano più tavole. $\mathrm{Da}$ ciò si ricava facilmente l'impressione di un'opera approssimativa e casuale; tuttavia Genelli riesce a dare una forma che produce unità artistica". 
magine. Le figure accucciate in primo piano formano insieme uno schema triangolare che punta in direzione del dimonio; gli sguardi di questi dannati, rivolti lateralmente, generano inoltre un movimento quasi a spirale verso l'alto, che termina sulla figura del nocchiero infernale. L'immagine sembra così dominata da un ritmo lento che è quello dei versi danteschi "come d'autunno si levan le foglie / l'una appresso de l'altra" (vv. II2-II3), mentre il braccio sollevato di Caronte "traduce" il gesto che il poeta gli attribuisce (v. IIo: "loro accennando"). Le espressioni del volto dei dannati, come pure i loro atteggiamenti, implicano, in accordo con il testo, terrore (v. IOI: "cangiar colore e dibattero i denti") ed ira (v. I03: "bestemmiavano Dio e lor parenti"). Osservando più attentamente si nota sullo sfondo, immediatamente a destra della mano di Caronte, la testa di un moro, che sembra quasi alludere al peccato come caratteristica universale dell'intera umanità senza distinzioni di popolazione o di appartenenza geografica.

Genelli si richiama in modo evidente al linguaggio figurativo del Michelangelo della Cappella Sistina, in particolare del Giudizio Universale. Negli anni trascorsi a Roma egli aveva avuto modo di studiare attentamente gli affreschi del grande maestro del Rinascimento, che egli non solo considerò per tutta la vita come esempio di disegno anatomico - come testimonia uno studio conservato nel Kupferstichkabinett di Berlino ${ }^{\mathrm{IO}}$ e ispirato per l'appunto al Giudizio - ma anche come repertorio di motivi e tipi iconografici. Un esempio è il dannato in secondo piano a sinistra, che guarda verso Caronte: questa figura ricorda il disperato peccatore che nell'affresco michelangiolesco viene portato via da un diavolo, ripreso in modo ancora più evidente, come vedremo, in uno dei successivi disegni (Tav. 9). Diversamente da Michelangelo, Genelli non attribuisce a Caronte tratti demoniaci, come del resto avevano fatto Flaxman e Koch; si spinge però ancora oltre, modellandone la figura di statuaria perfezione fisica ${ }^{\mathrm{II}}$ secondo i canoni della scultura classica; la testa mostruosa del nocchiero, così come essa compare nella Sistina, viene piuttosto ripresa nell'illustrazione a If. XXII ed attribuita ad un diavolo. L'influenza della scultura antica è caratteristica costante di tutte le illustrazioni alla Divina Commedia. Che Genelli lavorasse anche per reminiscenze e riprese d'immagini considerate come modelli tipologici, lo dimostra anche il dannato in piedi sullo sfondo, appoggiato all'albero della nave, il quale sembra modellato sullo schema classico della "statua loricata" romana.

Io. Inv. \#130.

II. Questo tipo iconografico di Caronte ritorna in successive rappresentazioni ottocentesche; un esempio da confrontare è la stampa di Bartolomeo Pinelli sull'episodio analogo contenuto nel sesto canto dell'Eneide. 
2. L'importanza della citazione come elemento chiave dello stile di Genelli ${ }^{12}$ è particolarmente chiara nell'illustrazione al quarto canto. La scena si trasferisce nel Limbo, dove gli spiriti magni del mondo pagano vivono in eterno e inappagato desiderio di Dio. Tuttavia, oggetto della rappresentazione non è l'episodio chiave del canto, e cioè l'incontro di Dante con la bella scola dei grandi poeti dell'antichità, quanto piuttosto la liberazione dei patriarchi dell'antico Testamento in occasione della discesa di Cristo agli Inferi, secondo la narrazione apocrifa del Vangelo di Nicodemo. La composizione è organizzata in modo simmetrico intorno alla figura di Cristo: Due gruppi, comprendenti ciascuno tre figure di salvati, stanno a destra e a sinistra del Salvatore e rivolgono su di lui i loro sguardi, mentre egli sale in cielo. In primo piano compaiono cinque personaggi maschili tra cui un poeta, riconoscibile dalla corona di alloro e presumibilmente identificabile con Virgilio quale osservatore e narratore della scena (If. IV, 53-55: "io era novo in questo stato / quando ci vidi venire un possente / di segno di vittoria coronato"). Il ruolo centrale di queste figure è sottolineato dal fatto che esse formano con quella di Cristo uno schema triangolare, evidenziato dal panneggio delle stoffe. In primo piano, dietro ad uno sperone roccioso, si apre un abisso. Questa strategia viene utilizzata in quasi tutti i disegni e rientra nel novero dei mezzi espressivi con cui l'artista trasmette il senso della profondità. Anche in questo caso egli si richiama ad una soluzione tipicamente michelangiolesca, adottata per esempio nella scena del Diluvio Universale della Cappella Sistina.

Adamo, Eva e Abele sono immediatamente riconoscibili dai loro attributi tradizionali. Lo stesso vale per Mosé, caratterizzato dalle corna luminose e dalle tavole della legge, per Davide, identificabile dalla corona, e per Abramo. La dipendenza di Genelli da Michelangelo è anche qui subito evidente: la raffigurazione di Mosé si riallaccia tipologicamente alla statua dell'incompiuta tomba di Giulio II, mentre quella di Eva ricorda la Creazione di Eva della Cappella Sistina, anche se l'immagine appare invertita in senso speculare rispetto al modello. Una ulteriore citazione si può riconoscere nella figura di Cristo, ispirata alla statua che lo scultore danese Bertel Thorvaldsen aveva creato nel i82I per la chiesa di Nostra Signora (Vor Frue Kirke) di Copenhagen: Quest'opera, all'epoca assai popolare in Germania, fu largamente imitata e servì da modello, tra l'altro, per la statua di Cristo della Friedenskirche di Potsdam.

3. L'immagine di Minosse come personificazione di inflessibile giustizia è al centro della terza illustrazione. Con una corona in testa, egli appare in margi-

I2. La combinazione di riferimenti a note opere d'arte dell'antichità, note a Genelli dagli anni romani o attraverso pubblicazioni di archeologia, è già caratteristica dei due cicli d'illustrazioni dedicati ai poemi omerici. 
ne a destra, seduto sui gradini di un trono di pietra, mentre uno dopo l'altro ascolta le confessioni dei peccatori, introdotti e subito dopo portati via da diavoli. La figura statuaria provvista di coda di serpente, le cui circonvoluzioni intorno al corpo indicano il girone infernale da assegnare ai peccatori, ricorda il Minosse della Sistina, ispirato comunque alla rappresentazione dantesca. Tuttavia, a differenza che in Dante (If. V, 4: "stavvi Minòs orribilmente, e ringhia”) e in Michelangelo, essa non presenta tratti demoniaci di particolare evidenza. Il Minosse di Genelli è un tipico esempio della sua tendenza a rappresentare le creature infernali come esempi di statuaria perfezione fisica, come già osservato a proposito di Caronte. Lo stesso vale per le figure dei diavoli accompagnatori, tra cui colpisce particolarmente quello in piedi in secondo piano accanto alla peccatrice, chiaramente modellato secondo il canone policleteo. Nel secondo diavolo, che trasporta una donna verso l'Inferno, si può riconoscere una reminiscenza del Giudizio Universale dipinto da Luca Signorelli nel Duomo di Orvieto.

Nel trasporre la scena dalla prospettiva del poeta-osservatore, Genelli conferisce particolare evidenza al motivo della giustizia, lasciando completamente in sottofondo altri elementi del testo dantesco. Mentre gli occhi di tutte le figure, concentrati su Minosse, ne sottolineano la funzione di protagonista della scena, la tensione emotiva si addensa nella comunicazione degli sguardi che unisce il giudice e la peccatrice. Attraverso il ricorso ai motivi del trono e della corona (particolare, questo, che ritorna in Doré), la messa in scena di Minosse si riallaccia al tipo del re-giudice ${ }^{13}$ e ne evidenzia pertanto l'atteggiamento piuttosto inquisitorio che minaccioso. In questo contesto, l'artista inserisce la figura del diavolo accompagnatore, che si cercherebbe invano in Dante, il cui sguardo rivolto alla peccatrice sembra esprimere una sorprendente commiserazione. Il rapporto creativo con il testo della Commedia si può osservare inoltre in altri dettagli dell'immagine, come la testa maschile con una mitria vescovile che appare sullo sfondo: con essa Genelli sembra alludere all'atteggiamento di Dante, spesso apertamente polemico, nei riguardi della chiesa.

Considerata nel suo rapporto con i due disegni immediatamente successivi, questa tavola si presenta come il capitolo d'apertura di un ciclo secondario di illustrazioni, dedicato al quinto canto della Divina Commedia in cui Dante incontra le anime dei lussuriosi. Il confronto con la quarta illustrazione consente di identificare i personaggi in piedi davanti a Minosse in attesa del giudizio con Paolo e Francesca. In ciò si riconosce un intervento personale dell'artista che evidenzia la continuità tra le diverse immagini e rafforza così

I3. Si veda, per citare solo un esempio, il Giudizio di Salomone (I533; Venezia, Gallerie dell'Accademia) del pittore veronese Bonifazio de’Pitati (I487-I553). 
la coerenza narrativa all'interno del ciclo. L'osservatore viene così preparato al tema della tavola successiva, che raffigura l'episodio forse più noto dell'intera Commedia.

4. Insieme con gli altri lussuriosi, Paolo Malatesta e Francesca da Rimini vengono trascinati dalla bufera quale contrappunto allegorico del vento delle passioni, da cui questi peccatori si sono lasciati trasportare in vita. Alcune delle figure storiche o letterarie nominate da Dante sono immediatamente riconoscibili o vengono in modo allusivo ricordate. La donna reclinata a sinistra, che reca una corona, in mancanza di altri più precisi attributi riassume probabilmente in sé le tre regine citate da Dante, Cleopatra, Didone e Semiramide; Paride (v. 67: "vedi Parìs") si riconosce a destra dal berretto frigio, che si richiama alla tradizionale iconografia di questo personaggio nell'arte greca; analogamente, l'acconciatura alla greca permette la possibile identificazione della seconda figura maschile con Achille "che con amore al fine combattéo" (v. 66). L'interpretazione di questa scena da parte di Genelli si iscrive a pieno titolo nella tradizione tedesca tardo-classicista di Carstens e Koch ${ }^{\mathrm{I4}}$. Da questi artisti, Genelli trae in particolare le figure delle divinità alate del vento, assenti in Dante: il poeta non fa cenno all'origine della bufera infernale, forse in relazione con lo sbattere delle ali di Lucifero che nel profondo Inferno determina la formazione del ghiaccio. La loro funzione originaria come personificazioni della bufera infernale ${ }^{\mathrm{IS}}$ tuttavia non viene ripresa, soprattutto perché il motivo del vento è appena accennato attraverso il movimento di capelli e stoffe. I demoni si trasformano piuttosto in immagini di lamento, il cui atteggiamento compassionevole riveste una importante funzione narrativa: da una parte esso rappresenta in modo figurato l'effetto patetico del discorso di Francesca, dall'altro allude al breve momento di pausa della bufera, che nel testo di Dante consente la conversazione del poeta con l'infelice coppia riminese (v. 93: "mentre che il vento, come fa, si tace"). Sul piano figurativo, il dialogo dei personaggi viene colto come comunicazione di sguardi e presentato attraverso la disposizione dei vari elementi della scena come momento centrale di essa. Nonostante Genelli si rifaccia a una struttura già presente nelle analoghe illustrazioni di Koch, egli evidenzia per mezzo della simmetria centrale in modo ancora più accentuato la relazione tra il poeta e la coppia di amanti. La composizione si basa sulla contrapposizione spaziale sopra-sotto ed è organizzata intorno alla scena principale, rappresentata al centro dell'immagine, secondo uno schema chiastico. La rappresentazione a tre quarti della figura di Dante è in questo contesto utilizzata per collocare la testa del poeta

I4. Sull'iconografia degli infelici amanti si veda Soennecken (2002).

15. In questa funzione, le figure dei demoni in Carstens e Koch si rifanno all'iconografia tradizionale del vento come figura con le guance gonfie intenta a soffiare. 
e quella di Francesca su una linea parallela e convogliare l'attenzione sul contatto dei loro sguardi. La posizione di Paolo, che con le braccia ed il mantello crea attorno a Dante e Francesca una sorta di cornice, ne sottolinea il ruolo di protagonisti: Paolo rappresenta in questo senso una reminiscenza del tipo classico del Discobolo, che Genelli, anche in altri contesti, utilizza come modello $^{16}$. La figura a tre quarti di Virgilio trasmette da una parte, nella funzione di repoussoir, il senso della profondità, dall'altra contribuisce a marcare la suddivisione simmetrica della scena evidenziando quanto raffigurato in secondo piano. Questo ruolo compete anche alle divinità del vento, i cui sguardi rivolti a Dante e Francesca guidano quello dell'osservatore. Da notare che Genelli, diversamente da Flaxman e dalla maggior parte degli illustratori, sceglie di non rappresentare lo svenimento di Dante (v. I42: "e caddi come corpo morto cade" ) mettendo piuttosto a fuoco la drammaticità del colloquio con Francesca: tale scelta consente inoltre di accentuare la continuità narrativa con la tavola successiva.

5. Tema del quinto disegno è la celebre scena del libro galeotto, in cui Paolo e Francesca sono indotti dalla lettura comune del Lancelot ou le conte dou Graal a riconoscere e confessarsi reciprocamente il loro amore. Genelli la interpreta secondo un modello iconografico assai popolare nel XIX secolo ed utilizzato, tra gli altri, da Ingres, Delacroix e Feuerbach: al centro dell'immagine appare la coppia immersa nella lettura, mentre Gianciotto Malatesta, in atto di estrarre il pugnale con cui ucciderà moglie e fratello, si introduce di soppiatto dietro alla tenda con l'intento di sorprenderli. Una certa vicinanza rispetto al disegno di Flaxman si mostra nella rinuncia a una raffigurazione dettagliata dello sfondo, che permette di concentrare l'attenzione sulle figure che agiscono nella scena. Questa acquista tuttavia una particolare intensità, dovuta ad uno scarto fondamentale rispetto alla maggioranza delle illustrazioni: come Delacroix, e, più tardi, Feuerbach, Genelli lascia completamente fuori il motivo del bacio. Questa scelta produce una accentuata tensione narrativa e coglie il punto cruciale del testo dantesco, cioè la scoperta dell'amore grazie al libro che agisce da catalizzatore: "Galeotto fu il libro e chi lo scrisse" (v. I37). La costruzione spaziale sottolinea in modo assai efficace questa prospettiva. Mentre i gradini e le colonne a margine dell'immagine creano l'illusione della profondità, la prevalenza delle linee verticali crea una sorta di cornice che isola ed evidenzia le figure di Paolo e di Francesca. Gli occhi degli amanti ed il libro nella mano di Paolo si uniscono a formare un triangolo che costituisce il contenuto centrale dell'immagine: Il panneggio dell'abito di Francesca, a sua volta ripreso dal movimento della tenda, sposta a sua volta l'attenzione verso

I6. Cf. Ebert (I97I: 68), la raffigurazione dell'anima di Patroclo nei Profili omerici (20). 
destra, in modo da guidare l'osservatore verso l'ingresso a sorpresa di Gianciotto. Il ventaglio a metà ripiegato di Francesca, che non compare in altri illustratori, rappresenta in questo contesto un elemento solo apparentemente marginale. Con esso, Genelli integra nella scena un motivo simbolicamente associato con l'amore e la sensualità, nel quale si cristallizza sul piano figurativo la tensione erotica dei protagonisti e il loro desiderio del bacio.

6. Anche l'episodio dell'ottavo canto raffigurato nella sesta tavola godeva nelle arti figurative di una notevole fortuna, di cui testimonia tra l'altro il noto dipinto di Delacroix La barque de Dante (I822). Dante e Virgilio sono qui rappresentati mentre sulla barca guidata dal demone Flegiàs attraversano il "brago" in cui affondano gli iracondi in direzione della città di Dite. In accordo con il testo, alcune torri appaiono a destra all'orizzonte attraverso il fumo della palude stigia (If. VIII, 70-7I: "le sue meschite / là entro certe ne lo vallo cerno"). La composizione, concepita in base a uno schema piramidale, si contraddistingue per il continuo incrociarsi dei piani e delle linee e per il sovrapporsi delle linee curve, con cui l'artista riesce efficacemente a rendere il senso del movimento e della confusione degli elementi. La calma dei poeti al centro dell' immagine forma un netto contrasto con la brutalità bestiale dei dannati che lottano l'uno con l'altro: al centro dell'immagine sembra essere, più che la resa dettagliata degli accadimenti narrati, il momento in cui Dante medita sulle conseguenze dell'ira e dell'orgoglio. In questo senso, una figura non identificabile con corona, che Genelli introduce in modo apparentemente arbitrario rispetto al testo, non fa che identificarne ed estrapolarne un dettaglio riassumendo simbolicamente il contenuto della riflessione di Dante tramite l'allusione al motivo della regalità: il particolare traduce in immagine l'osservazione di Virgilio sul peccato dell'orgoglio (vv. 48-5I: "Quanti si tengon or là su gran regi / che qui staranno come porci in brago, / di sé lasciando orribili dispregi!").

Dal testo apprendiamo come il fiorentino Filippo Argenti cerchi di appendersi alla barca e venga respinto indietro da Virgilio. Tuttavia, né questa scena, né il breve scambio di battute tra Dante e l'iracondo sono oggetto dell'illustrazione, quanto piuttosto il momento immediatamente successivo, quando tutti i dannati si gettano su Argenti mentre questi rabbiosamente morde se stesso (vv. 6I-63: "Tutti gridavano: A Filippo Argenti! / e il fiorentino spirito bizzarro / in sé medesmo si volgea co'denti"). Filippo Argenti è riconoscibile nel nudo di spalle in primo piano, respinto dagli altri nell'acqua a suon di botte. L'osservatore non può vedere, ma solo immaginare, il rabbioso autolesionismo del fiorentino spirito bizzarro, appena accennato nel ripiegarsi del braccio sulla faccia, con cui Filippo si difende dai colpi degli altri dannati. Eppure il tema dei denti è ripreso in altro modo e diventa un motivo 
centrale della tavola, che esprime in modo emblematico il tema del canto ${ }^{17}$ : diversamente dalle altre tavole, la resa dei sentimenti è qui affidata non solo agli occhi, ma anche e soprattutto alla bocca: la maggior parte delle figure, anche quelle sullo sfondo, hanno bocca aperta e denti in evidenza, così che il motivo del mordere ne emerge come espressione di ira selvaggia.

7. Un procedimento che mette insieme elementi provenienti da diversi luoghi del testo è utilizzato per rappresentare l'incontro di Dante con Farinata degli Uberti nella settima tavola. Il disegno segue da vicino il testo del decimo canto dell'Inferno: il poeta e il ghibellino, il cui braccio puntato verso Dante allude alla predizione dell'esilio, occupano il centro della scena, mentre l'ombra di Cavalcante de'Cavalcanti si erge dal suo avello per chiedere notizie sul destino del figlio Guido. Il paesaggio riproduce fedelmente la descrizione di If. IX, II2-I2I: "sì come ad Arli, ove Rodano stagna, / [...] fanno i sepulcri tutto il loco varo / così facevano quivi da ogni parte / [...] ché tra gli avelli fiamme erano sparte / per le quali eran sì del tutto accesi, / che ferro più non chiede verun' arte. / Tutti li lor coperchi eran sospesi”. In questo contesto, Genelli cerca di includere nell'immagine dettagli quali l'accenno da parte di Farinata a Federico II di Svevia quale peccatore punito nello stesso girone: "Qui con più di mille giaccio; / qua dentro è il secondo Federico, / e'l Cardinale; e de li altri mi taccio" (vv. II8-I2o). A questo si riferisce l'iscrizione Federigo II imperatore guardo, visibile sul coperchio del sarcofago sullo sfondo, la cui funzione affatto secondaria è segnalata dalla posizione centrale. La trasposizione figurativa si serve a questo scopo della sovrapposizione di due diversi luoghi danteschi: l'illustratore innesta sul discorso di Farinata la reminiscenza a un passo del canto successivo, in cui una iscrizione analoga compare in riferimento a papa Anastasio II (If. XI, 6-9: "ci raccostammo dietro ad un coperchio / d'un grande avello, ov'io vidi una scritta / che diceva: Anastasio papa guardo / lo qual trasse Fotin de la via dritta.").

La resa pittorica della figura di Farinata è anch'essa fedele alla descrizione dantesca: "Vedi là Farinata che s'è dritto; / da la cintola in su tutto il vedrai / [...] ed ei s'ergea col petto e con la fronte / como avesse lo 'nferno in gran dispitto" (If. X, 32-36). La fierezza del ghibellino si manifesta in senso idealizzante-eroizzante nell'evidenza dei dettagli anatomici: Genelli ne fa una figura statuaria modellata sull'esempio dei guerrieri dell'antica Grecia ${ }^{18}$. Il particolare delle sopracciglia sollevate si riallaccia invece all'accenno del testo

17. Il gesto autolesionista del rivolgersi “in sé medesmo [...] co' denti” è comunque presente nell'illustrazione e viene attribuito, invece che a Filippo Argenti, alla figura dell'uomo col pugno alzato a destra.

I8. Secondo Eberts (197I: 70) il torso di Farinata sarebbe esemplato su quello di "un Laocoonte". 
all'espressione del viso (v. 45: "ond'ei levò le ciglia in poco in suso"). Anche in questo caso, l'intensità del dialogo con Dante viene trasmessa in primo luogo dal contatto degli sguardi; la diretta relazione tra il poeta e Farinata viene poi sottolineata dalla struttura della composizione, che colloca le due teste su una diagonale, mentre il panneggio del lenzuolo intorno al corpo del ghibellino, proseguendo nelle pieghe del mantello di Dante, incornicia le figure. Questo dettaglio, che richiama il motivo del sudario e che Genelli trovava già in Flaxman, compare con la stessa funzione compositiva nell'analoga illustrazione di Doré.

8. Dopo l'incontro con Farinata, la tavola successiva passa ad illustrare il dodicesimo canto, mostrando Dante in atto di attraversare il Flegetonte in groppa al centauro Nesso.

Dal punto di vista compositivo, il quadro si caratterizza per il ritmo ascendente e per il divergere delle linee. La linea curva che iniziando dal nudo di spalle prosegue nelle teste del dannato con corona e dei centauri sullo sfondo suggerisce all'occhio dell'osservatore, come avviene nelle tavole precedentemente esaminate, una traccia da percorrere e mette in evidenza l'associazione simbolico-allegorica di queste creature mitologiche con la tirannide: ci troviamo infatti nel settimo girone, dove violenti e tiranni sono tormentati dalle frecce dei centauri arcieri. Genelli sembra avere rinunciato alla resa dettagliata del testo per quanto riguarda le figure storiche menzionate da Dante: nessuno dei dannati, ad esempio, è immediatamente identificabile come Guido da Monforte quale "ombra da l'un canto sola" (If. XII, II8), a meno che non si pensi ad un effettivo consapevole scarto dell'artista rispetto alla descrizione dantesca, in cui questo personaggio fa parte di quelli che affondano nel lago di sangue fino alla gola (vv. II6-II7: "sovr'una gente che fino alla gola / parea che di quel brulicame uscisse"). La corona regale o l'elmo costituiscono piuttosto degli elementi di riconoscimento con carattere esemplare. Da notare la evidentissima ripresa michelangiolesca nella figura del re seduto, che riproduce, seppure con molta meno drammaticità, uno dei più noti dannati del Giudizio Universale.

Il torso statuario su corpo equino dei Centauri e le orecchie a punta che ne evidenziano la natura ferina richiamano invece l'iconografia tradizionale di queste figure nella scultura classica ${ }^{19}$. Si riconosce agevolmente Chirone dalla posizione della testa, che ricorda If. XI, 70-7I: "quel di mezzo, ch'al petto si mira".

Nesso è invece raffigurato in primo piano, con in groppa Dante e in atto di parlare con lui. Il nudo di spalle, ergendosi dal lago di sangue verso i pro-

19. Cf. Come esempio le statue di centauro dei musei Capitolini. 
tagonisti, crea un movimento ascendente verso di lui e ne evidenzia il dialogo con il poeta, questa volta reso attraverso il contatto tra le teste, che insieme con quella di Virgilio formano il ricorrente schema triangolare. Una funzione di raccordo col testo è affidata al contrasto tra la figura di Dante seduto e quella di Virgilio, rappresentato, come mostra la posizione delle gambe e il panneggio del manto, in volo: L'allusione è ai versi in cui il poeta chiede a Chirone un accompagnatore per Dante, che non sarebbe in grado da solo di attraversare il fiume bollente in quanto "non è spirto che per l'aere vada" (If. XII, 95).

9. Con la nona tavola inizia un secondo "ciclo nel ciclo", comprendente nel complesso quattro disegni e dedicato a Malebolge, che illustra alcuni episodi narrati da Dante nel ventunesimo e nel ventiduesimo canto dell'Inferno. Questa parte del poema doveva risultare per Genelli di particolare interesse, a causa del suo ritmo movimentato e della ricchezza di figure di contorno, caratteristiche queste che incontravano le preferenze artistiche dell'illustratore. Il primo episodio si situa nella quinta bolgia, dove i barattieri scontano la loro pena immersi nella pece bollente. Dalla prospettiva di Dante osservatore viene rappresentata la scena in cui un dannato, più tardi designato come uno dei magistrati al governo di Lucca (If. XXI, 38: "gli anzian di santa Zita"), è agguantato da un diavolo per la caviglia e scaraventato nella pece, dove lo attendono altri diavoli armati di uncini. L'illustrazione mostra in modo significativo come la tecnica del contorno non comporti necessariamente staticità o piattezza e sia interpretata da Genelli in modo assai diverso da quello di Flaxman. Fattori fondamentali per la dinamicità e la tensione drammatica della scena sono il movimento ascendente dato dalla curva che, partendo dal braccio del diavolo in primo piano, prosegue con le teste degli altri personaggi, oltre che dal gioco di linee incrociate e dalla messa a fuoco della torsione muscolare. Tutto ciò si accompagna a una disposizione armonica della composizione, in cui il corpo del diavolo stesso forma uno schema chiastico e sottolinea la simmetria dello spazio rappresentato. Anche questa figura è modellata secondo i canoni classici e ricorda tra l'altro l'iconografia tradizionale di Eracle con la clava ${ }^{20}$ : la sua natura demoniaca e bestiale si manifesta soltanto negli artigli delle mani e dei piedi, il cui straniante contrasto con le ali piumate richiama il motivo dell'angelo caduto. Il ricorso al modello michelangiolesco è infine evidente nel volto del demonio, a cui sono attribuiti i tratti mostruosi del Caronte del Giudizio.

20. Per un esempio cf. la raffigurazione di Eracle in Antonio del Pollaiolo. 
Io. Nella decima illustrazione, Genelli cerca di concentrare in un'immagine unica la complessa narrazione del canto XXII. Il diavolo Malacoda impone il silenzio agli altri demoni per consentire ai poeti di proseguire il loro viaggio e ordina a dieci compagni di accompagnarli lungo la bolgia. Il nudo di spalle in primo piano, a cui Malacoda sembra rivolgersi, è quindi da identificare con lo Scarmiglione del testo dantesco (vv. IO3-IO5: "quel demonio che tenea sermone / col duca mio, si volse tutto presto / e disse: Posa, posa, Scarmiglione!"). Motivo dominante della scena è però soprattutto la paura di Dante, concentrata nell'espressione degli occhi e nella posizione del corpo: a questo proposito, l'immagine riproduce da vicino il testo dei versi 97-99 ('I' m'accostai con tutta la persona / lungo , I mio duca, e non torceva li occhi / da la sembianza lor ch'era non bona"). A ciò contribuiscono i principi compositivi: il drappeggio dell'abito, i bastoni uncinati dei demoni e le diagonali formate dalle teste dei personaggi costituiscono una serie di linee che puntano verso il margine, esprimendo così la tensione verso la fuga. La gamba di Dante, tesa nel passo sotto al mantello, costituisce in rapporto a Malacoda una sorta di piedistallo che in associazione con le ali spiegate ne evidenzia la figura ed i gesti ${ }^{21}$.

Dal punto di vista iconografico ci troviamo ancora una volta di fronte a una varietà di citazioni. I grotteschi volti dei diavoli sono ispirati agli affreschi michelangioleschi della Sistina. Inoltre Genelli recupera alcuni ben noti topoi dell'arte antica, quali le gambe in forma di serpente del diavolo in basso a sinistra ed i serpenti sulla testa di Malacoda, che ricordano la figura mitologica di Medusa. La posizione eretta del demone alato si può considerare tra l'altro una reminiscenza dell'iconografia di Hermes con cadùceo: Malacoda appare in questo senso in un ruolo che ricorda, pervertendolo, quello tradizionale dello Psicopompo, l'accompagnatore delle anime ${ }^{22}$.

II. L'undicesima tavola rappresenta una scena dal ventiduesimo canto a cui anche Joseph Anton Koch aveva dedicato una delle sue illustrazioni. In primo piano sta il diavolo Graffiacane, nell'atto di tirare su col "ronciglio" un peccatore dalla pece bollente; questo, a domanda di Virgilio, si fa riconoscere come un servo del re di Navarra (identificato dai commentatori come Ciampolo) e racconta la sua storia. La scena è rappresentata così come Dante la vede, probabilmente ancora prima che questi chieda a Virgilio "fa, se tu puoi, che tu sappi / chi è lo sciagurato" (If. XXII, 43-44). L'artista trova ispirazione, più

2I. L'effetto prodotto ricorda la funzione analoga della conchiglia nella Nascita di Venere di Sandro Botticelli.

22. È possibile che Genelli considerasse la figura qui rappresentata nel ruolo di Malacoda come un motivo tipo, il cui effetto egli provava in diversi contesti. Suggerisce questa idea una delle tavole conservate a Weimar, in cui la stessa immagine viene inserita in una scena diversa insieme ai demoni in lotta di If. XXII. 
che nel dialogo con Ciampolo, nella resa dell'ambiente infernale e nella caratterizzazione delle varie figure di demoni ghignanti. La struttura compositiva dell'immagine si basa sulla contrapposizione quasi speculare tra demoni e dannati, che corrisponde alla ripartizione spaziale sotto-sopra. Il numero dei diavoli raffigurati si riferisce alla "decina" che Malacoda, nel canto precedente, assegna ai poeti come scorta; le loro ali piumate si riallacciano alla caratterizzazione, frequente nella Commedia, del demone come "malvagio uccello" (cf. If. XXII, 29) ${ }^{23}$. Mentre il volto del diavolo dal braccio teso è esemplato sul modello iconografico di Sileno, in quello in basso a destra è riconoscibile dalle zanne di cinghiale Ciriatto (cf. If. XXI, I22: "sannuto"; 22, 55-56 "a cui di bocca uscìa / d'ogni parte una sanna come a porco"). Nel disegno, la testa è posizionata in modo tale da evidenziare una delle due zanne; questo accorgimento evidenzia a come, nel testo, Ciriatto stia per utilizzarla per dilaniare il dannato (v. 57: "li fé sentir como l'una sdrucìa").

I2. Al seguito dell'episodio è dedicata la dodicesima tavola, con cui il ciclo secondario dedicato a Malebolge si conclude. Il soggetto è la lotta che si scatena tra i diavoli Calcabrina ed Alichino dopo che Ciampolo di Navarra è riuscito con uno stratagemma a sfuggire loro di mano e finisce con la caduta di entrambi nella pece bollente, mentre gli altri demoni assistono divertiti alla scena. Per la sua interpretazione pittorica, Genelli attinge alla metafora dell'uccello rapace, largamente dominante nel testo. Il concitato movimento di inseguimento e fuga dei demoni viene reso conferendo particolare evidenza ai piedi artigliati dei diavoli, in allusione ai motivi dello sparviero e del ghermire, caratteristici specialmente dei versi I38-I4I: "Ma l'altro fu bene sparvier grifagno / ad artigliar ben lui, e amendue / cadder nel mezzo del bogliente stagno". Il rapporto quasi speculare tra le ali ed i corpi delle figure in lotta e le loro braccia distese in parallelo riflettono la reciprocità dei gesti, di notevole rilievo nel testo dantesco (cf. If. XXII, I34: "dietro li tenne"; I38: "e fu con lui [...] ghermito"; I39-I40: "ma l'altro [...] ad artigliar ben lui").

I3. In netto contrasto con il ritmo dinamico di questa composizione sta la tredicesima tavola. Lenti e pesanti, sfilano davanti a Dante e Virgilio gli ipocriti, passando sul corpo nudo di Caifa crocifisso in terra (If. XXIII, 58-I20). I lineamenti del sommo sacerdote considerato responsabile della condanna di Cristo, deformati dal dolore, sono un diretto riferimento alla descrizione dantesca (v. II2: "quando mi vide, tutto si distorse"). Il tema centrale è costituito dal dialogo di Dante con i due Frati Gaudenti bolognesi: lo sforzo dell'artista è tutto nel rendere il motivo cardine del carico e del peso già evidente

23. Cf. anche $P d$. 29 , 118. 
nel ritmo lento del dettato poetico (cf. v. 67 "o in etterno faticoso manto!"), così come Dante lo rappresenta attraverso le cappe monacali dorate ma fatte di piombo puro. L'illustrazione si distingue infatti dalle altre per la drastica riduzione delle linee curve, come pure dei volti e delle nuvole; il senso del peso è efficacemente espresso attraverso il prevalere delle linee verticali ed il coincidere delle figure in primo piano in un blocco unico che campeggia al centro dell'immagine, creando, in rapporto al corpo crocifisso di Caifa, un senso di staticità.

I4. Il ciclo dedicato all'Inferno si conclude con altri due episodi tra i più celebri dell'intera cantica. La quattordicesima illustrazione mostra un diavolo nell'atto di sottrarre a san Francesco l'anima di un monaco del suo Ordine. Si tratta di Guido da Montefeltro, morto in abito da francescano e ciò nonostante punito tra i consiglieri fraudolenti per essersi lasciato comunque coinvolgere, ancorché monaco, in questioni politiche, fornendo a papa Bonifacio VIII suggerimenti per sconfiggere i suoi nemici in cambio di un'assoluzione preventiva. L'illustrazione si riferisce al racconto di Guido nel canto ventisettesimo dell'Inferno, che allude alla disputa tra Francesco e il diavolo per la sua anima, rappresentandone il momento finale, in cui il santo abbandona la contesa e ritorna in cielo. Ad un confronto dell'interpretazione di Genelli con i disegni di Joseph Anton Koch sullo stesso episodio, eseguiti nel I803, risalta la drastica riduzione dello sfondo ai contorni appena accennati della stanza. In tutte le sue versioni, l'interpretazione di Koch un lato evidenzia l'ambientazione storica della scena attraverso la cura dei particolari di sfondo, dall'altro ne accentua l'elemento drammatico, inserendo nell'immagine un corteo di monaci salmodianti e figure di angeli. Genelli si limita invece alle tre figure principali, collegate l'una all'altra attraverso strategie compositive. Con il loro movimento verso l'alto, i piedi sollevati di Guido accompagnano lo sguardo dell'osservatore verso Francesco che si solleva in volo; una funzione analoga assumono le pieghe dei sai monacali, che quasi si fondono l'uno con l'altro ${ }^{24}$. Le ali del diavolo formano poi rispetto a queste linee curve un contrappunto simmetrico, riportando l'attenzione sul corpo di Guido giacente a terra ${ }^{25}$.

La posizione del capo di Francesco, rivolto all'indietro, rinvia alla contesa con il demonio, appena terminata. Il sorriso maligno di costui, quale riferimento implicito al testo (If. XXVII, II4-I23) traduce in immagine la tagliente ironia con cui egli, nei versi danteschi, è raffigurato in veste di loyco, metten-

24. Dell'attenzione di Genelli per questi aspetti testimoniano i suoi numerosissimi studi di drappeggio, molti dei quali sono conservati nella Collezione Grafica della Fondazione Anna Amalia di Weimar.

25. La figura di Guido da Montefeltro giacente sulla stuoia fa da modello per la scena della Morte di Francesco d'Assisi, che Genelli rappresenta nella sua illustrazione al canto undicesimo del Paradiso. 
do in evidenza la contraddizione tra assoluzione e mancanza di pentimento come condizione necessaria per essa, che condanna Guido da Montefeltro alla dannazione (vv. I22-I23: “Forse / tu non pensavi ch'io loyco fossi!").

I5. La massiccia figura di Bertran de Born, che Dante incontra nel ventottesimo canto tra i seminatori di discordia, campeggia al centro della quindicesima illustrazione. Punito secondo il principio del contrapasso con la mutilazione del corpo, il poeta provenzale, assai stimato da Dante per i suoi versi, gli si presenta con in mano la propria testa mozzata "a guisa di lanterna" (If. XXVIII, I22).

I principi compositivi del disegno si riallacciano ad uno schema già presente in Flaxman, collocando Bertran quale personaggio principale al centro del'immagine. Paragonata con il contorno di Flaxman, tuttavia, la composizione acquista una dinamicità notevolmente maggiore. A questo scopo, Genelli si serve della dettagliata resa anatomica della figura del trovatore, raffigurato di spalle, che mette in risalto la plasticità delle forme. Il profilo dello sperone roccioso e il ponte sullo sfondo, su cui Dante e Virgilio osservano impressionati la raccapricciante visione, generano due superfici parallele sul piano orizzontale e suddividendo in questo modo lo spazio in settori nettamente definiti, cosa che conferisce ancora maggiore evidenza alla figura di Bertran. Mentre lo sporgersi di Dante dal ponte rafforza il senso della profondità, il contrasto tra il diavolo in volo verso sinistra e la schiera dei dannati in cammino verso destra accentuano l'impressione del movimento.

Il diavolo armato, raffigurato di tre quarti, corrisponde in generale alla descrizione di Dante (vv. 36-39: "un diavolo è qua dietro che n'accisma / sì crudelmente, al taglio de la spada / rimettendo ciascun di questa risma"); la spada e le ali piumate, in associazione con i tipici elementi identificativi della natura demoniaca (la coda e la testa anguicrinita) ne fanno una sorta di rovesciamento-perversione della figura di Michele arcangelo ${ }^{26}$. Numerose reminiscenze a noti motivi del'arte classica si possono riconoscere nell'intera illustrazione. In particolare, la posizione del torso di Bertran reggente la testa ricorda il tradizionale tipo del Perseo con la testa di Medusa: il ricorso a questo modello è molto antico e si può osservare anche in Botticelli ${ }^{27}$. La raffigurazione del dannato Bertran nella posizione caratteristica di Perseo dà

26. Nella tavola analoga conservata a Weimar, il diavolo compare con gli occhi chiusi e la bocca meno aperta.

27. Il pittore fiorentino ricorre alla tradizione iconografica del Perseo per rappresentare non solo Bertran de Born nel disegno su If. XXVIII, ma anche la figura del diavolo che in If. IX urla "Vegna Medusa: sì 'l farem di smalto!" (v. 52). Barbara Watts ha osservato come l'attribuzione congiunta a Bertran e a un demonio del gesto "eroico" di Perseo da parte di Botticelli generi una certa ambivalenza. sembra ricordare che il suo "eroismo" e la sua fierezza non sono meno ridicoli dell'atteggiamento qdel diavolo, avendogli fruttato la condanna eterna. 
luogo in Genelli come in Botticelli ad uno stridente e quasi ironico contrasto, in quanto il gesto trionfante dell'eroe greco che sconfiggendo Medusa riporta l'ordine e la pace si svuota di senso, trasformandosi nell'inutile appello di un seminatore di discordie, che nel corso della sua vita li ha minati alla base.

16. La crudele morte del conte Ugolino della Gherardesca, in cui l'elemento tragico nella Divina Commedia raggiunge il suo culmine, è il tema dell'ultima tavola dell'Inferno. La storia del podestà ghibellino di Pisa imprigionato per tradimento con due figli e due nipoti e lasciato morire di fame nella Torre de' Gualandi, narrata nel trentatreesimo canto, era divenuta già nel XVIII seco$1 \mathrm{l}^{28}$ popolare per il suo alto contenuto drammatico, trovando non solo nelle arti figurative grande diffusione.

Nel rappresentare l'episodio di Ugolino, Genelli prende manifestamente le distanze da tutta una tradizione che attraverso la resa dettagliata di architetture ed abiti tendeva ad inquadrare la scena in un preciso contesto storico. Ugolino e i suoi figli e nipoti, immaginati in età quasi infantile secondo la versione poetica di Dante, appaiono nudi. Lambiente di sfondo, come nella tavola di Guido da Montefeltro appena schizzato, si caratterizza come prigione solo grazie al motivo dell'anello con catena, visibile sul muro in basso a sinistra, che funge da elemento pars pro toto; inoltre, nessuna delle figure mostra tracce fisiche dei tormenti della fame, a cui sembra accennare solo la mano di Ugolino appoggiata sullo stomaco. Privata dei suoi elementi realitici, la scena acquista così un carattere a-storico ed esemplare.

Due esseri infernali attendono l'anima di Ugolino per trascinarla nell'abisso. Mentre il personaggio femminile si presenta con i tratti di una Furia, in quello maschile è stato più volte riconosciuto un vampiro ${ }^{29}$. Questa interpretazione è però dubbia alla luce del confronto con le illustrazioni dedicate a Malebolge, che mostra come i denti appuntiti e le ali da pipistrello rientrino, nell'immaginario di Genelli, tra gli attributi dei diavoli in generale ${ }^{30}$. Tenuto conto di ciò, la figura che qui compare si può identificare come un ennesimo demone che accorre a prelevare un'anima destinata alla dannazione. La Furia invece, elemento forse ripreso da un bassorilievo cinquecentesco dello scultore fiorentino Pierino da Vinci raffigurante la storia di Ugolino ${ }^{31}$, riveste una centrale funzione simbolica legata al tema del tradimento e collegata al ruolo di questa figura nell'antica mitologia greca, in cui l'Erinys simboleggia la vendetta per i delitti contro i parenti. La tradizione imputava infatti al

28. Cf. il dipinto di Sir Joshua Reynolds (Ugolino and his Children in the Dungeon, 1773).

29. Così Ebert (196I-62), Crass (I98I), Hempel (1954).

30. Si veda la tavola I2 con Ciampolo di Navarra, la lotta dei demoni nella tavola I3 come pure il demone protagonista dell'episodio di Guido da Montefeltro.

3I. L'opera risale al I548-I549 ed è conservata al Museo Nazionale del Bargello. Ne esistono diverse copie. 
conte non solo la consegna di alcune roccaforti pisane in mano nemica, ma anche di avere congiurato con la parte guelfa ai danni del nipote Nino, a lui associato al governo di Pisa. In questo contesto, la presenza della Furia allude allegoricamente alla molteplice colpa del conte, che appariva, agli occhi di Dante, traditore sia della patria che della famiglia.

\section{VI}

In conclusione, un'analisi dettagliata delle illustrazioni indica nel continuo ricorso ai modelli iconografici dell'antichità classica, nella tensione verso una rappresentazione estremamente austera con pochi dettagli di sfondo o di contorno, nella resa tutto sommato assai controllata di passioni e sentimenti le tendenze fondamentali dell'approccio di Genelli al testo dantesco. Si tratta di caratteristiche strettamente connesse con i principi del classicismo puro seguiti dall'artista, presenti nell'intera sua opera grafica e pittorica, ma ancora più evidenti in relazione ad un soggetto come la Divina Commedia. Se la tecnica del profilo quale forma privilegiata di rappresentazione può far apparire l'artista, almeno ad un primo sguardo, un seguace di John Flaxman, un'osservazione più accurata rivela come l'analogia sia in realtà valida solo in misura limitata. Nella sua trasposizione figurativa del mondo di Dante, Genelli si distanzia notevolmente dal linguaggio formale di Flaxman, orientato soprattutto alla pittura vascolare antica ed al bassorilievo. Lo sforzo dell'artista tende in primo luogo a sfruttare tutte le possibilità espressive consentite dalla superficie bidimensionale, cercando quasi di superarne i limiti in direzione della resa plastica delle forme e dell'espressione della profondità. A tale scopo, egli utilizza l'incrociarsi ed il sovrapporsi delle linee create da nuvole, onde, capelli, tessuti per esprimere sullo spazio dell'immagine la confusione degli elementi naturali, l'infuriare delle fiamme e l'intensità dei momenti emozionali e psicologici di volta in volta rappresentati. Inoltre, tramite il ri-uso del repertorio iconografico dell'arte classica, l'Inferno dantesco viene staccato dal suo specifico contesto cristiano e interpretato essenzialmente come un racconto mitologico, anche grazie alla rinuncia ad una raffigurazione dettagliata degli eventi, che li astrae in parte dal loro contesto lasciando abbondante spazio all'immaginazione dell'osservatore. In accordo con il principio teorico della "disposizione poetica dell'artista" quale fondamento dell'arte di Bonaventura Genelli, la semplicità lineare dei Contorni si rivela quindi strumento per un effettivo Wiederdichten, costituendo il pendant pittorico del diretto e pure altamente espressivo linguaggio poetico in cui prende forma l'immaginario dantesco. 

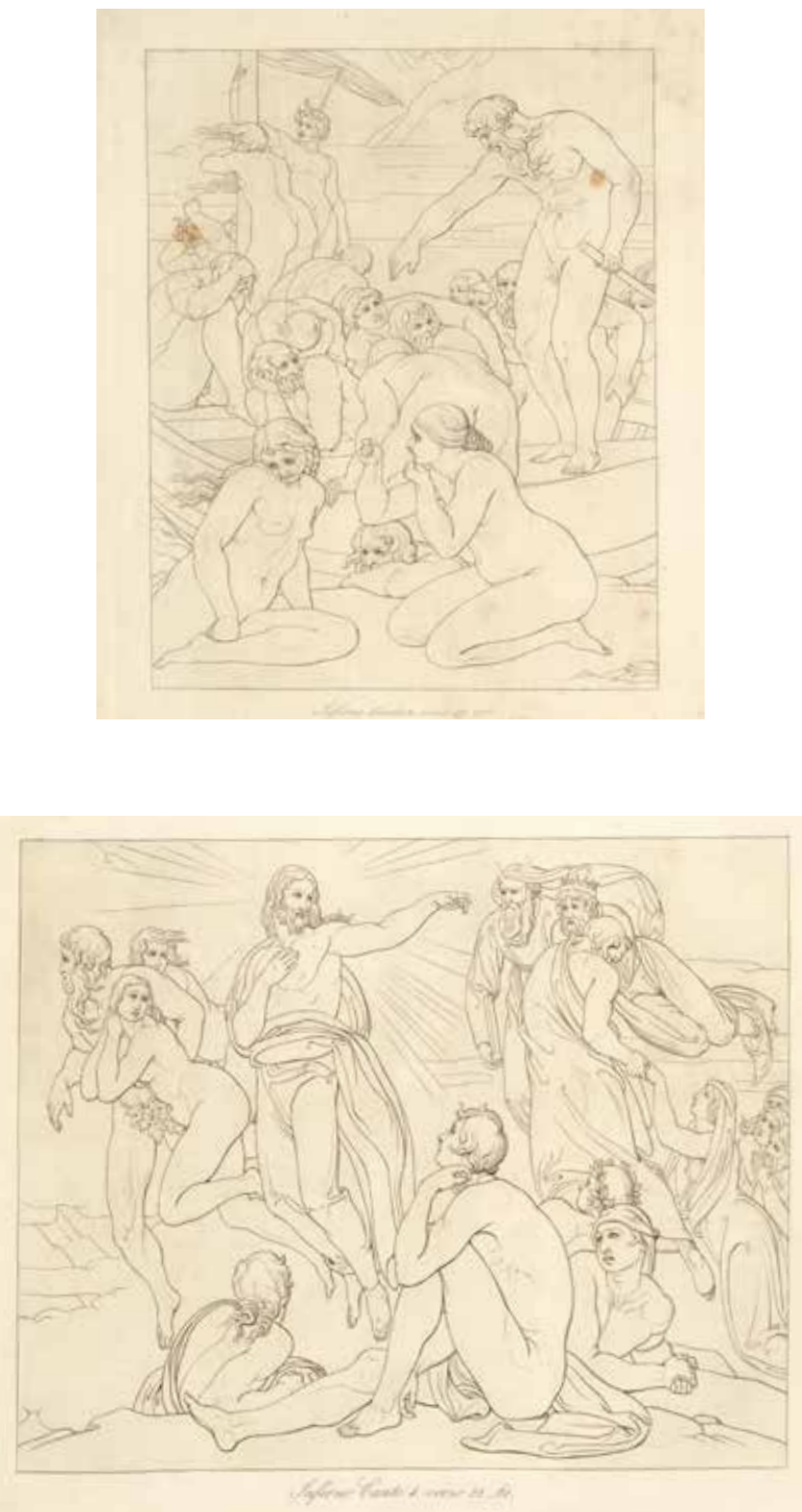

Immagini tratte da: Buonaventura Genellis Umrisse zu Dantes Goettliche Komoedie, hrsg. von Max Jordan, Leipzig: Duerr 1867.

Fig. I: Inferno III, vv. 7-I2O.

Fig. 2: Inferno IV, vv. 52-6I. 

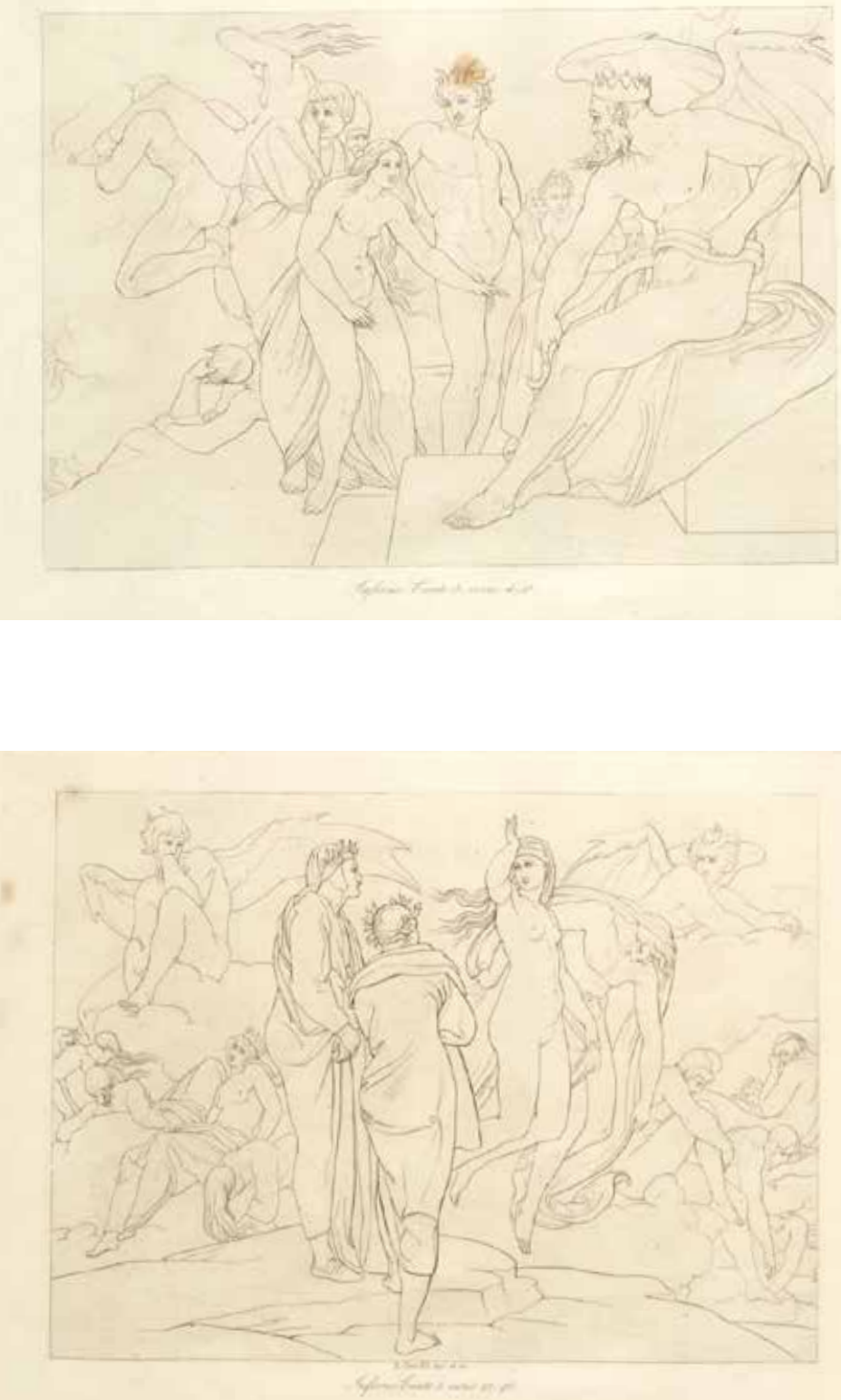

Fig. 3: Inferno V, vv. 4-I5.

Fig. 4: Inferno V, vv. 28-96. 

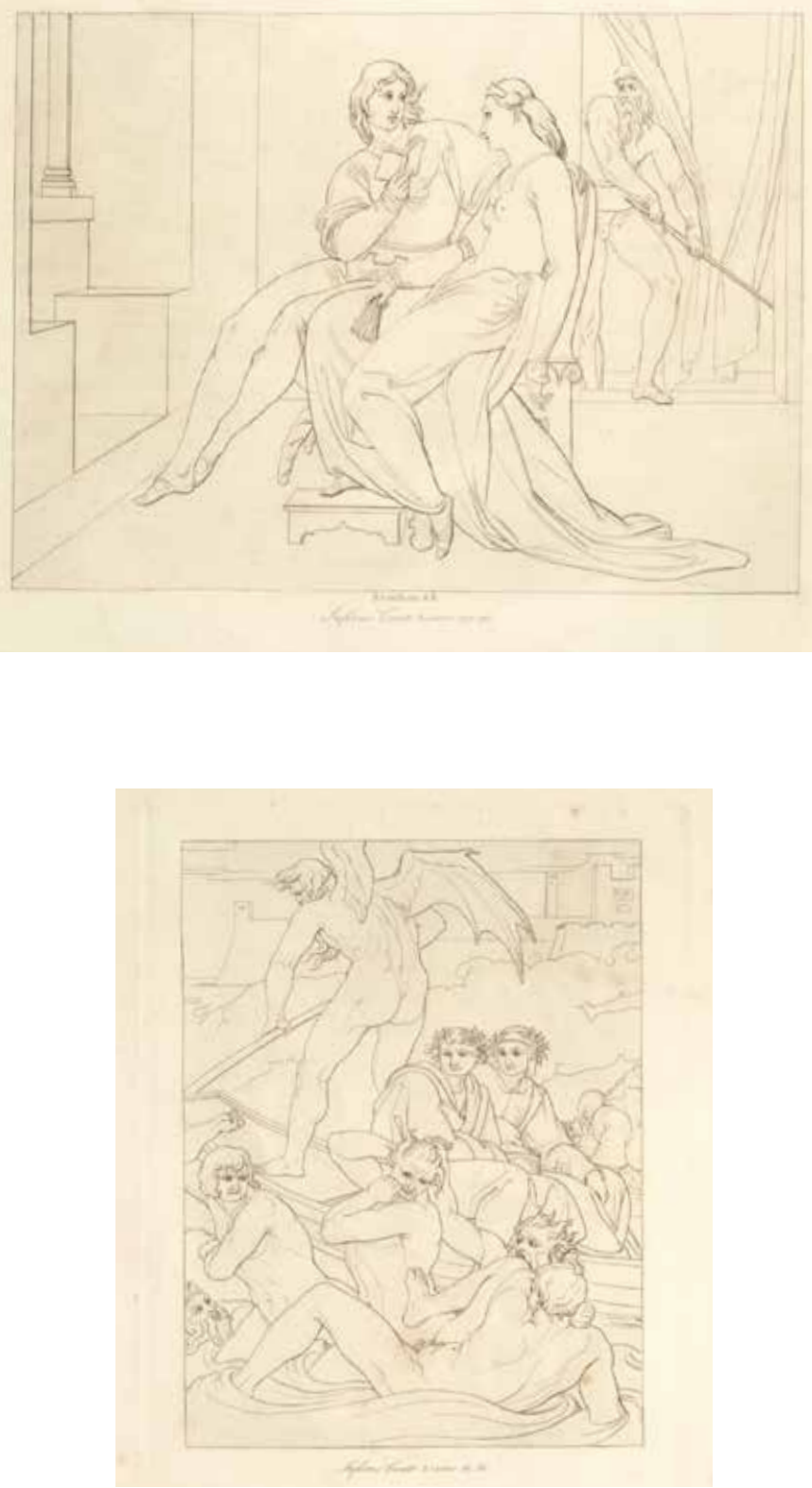

Fig. 5: Inferno V, vv. I27-I3I.

Fig. 6: Inferno VIII, vv. I3-63. 


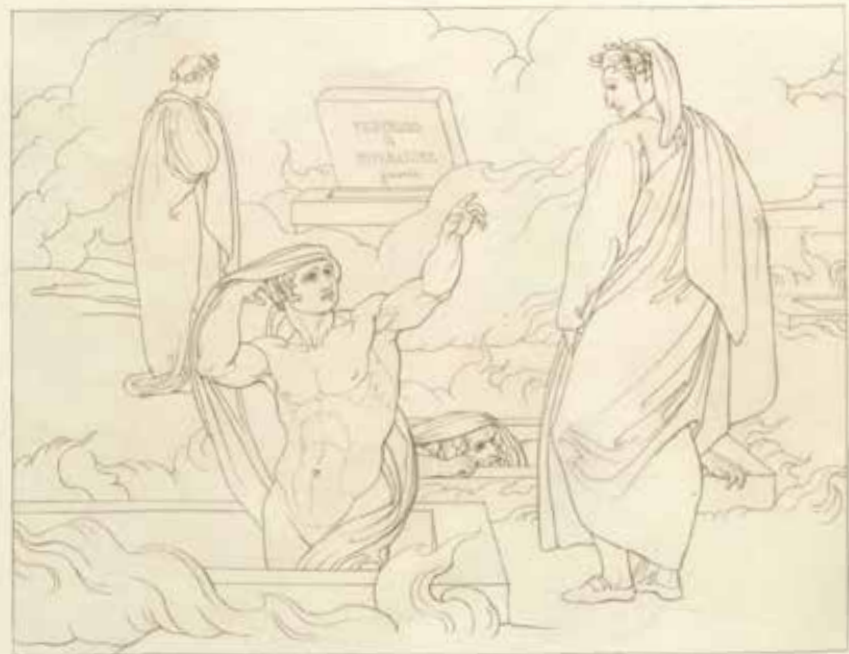

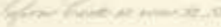

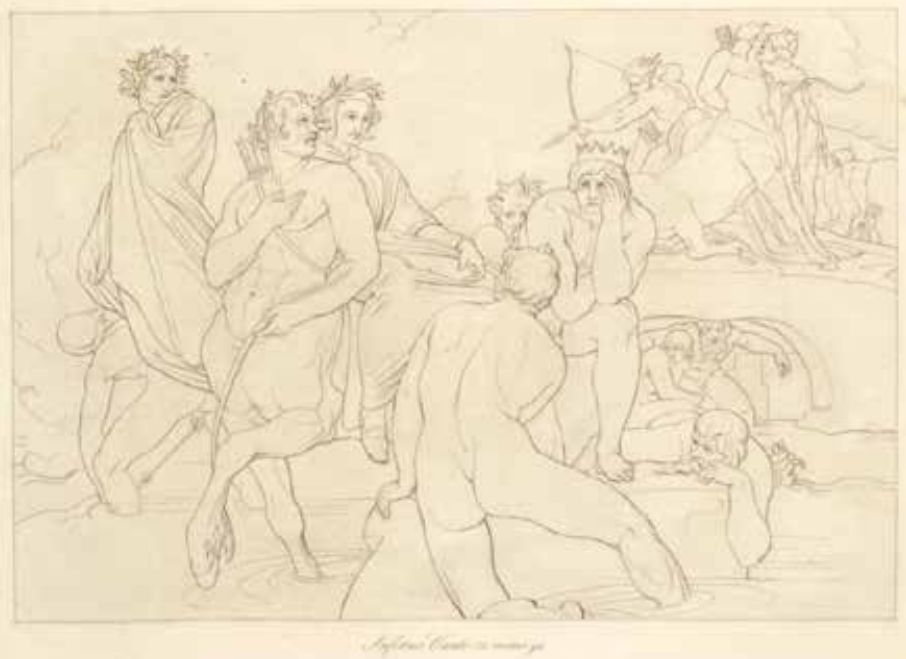

Fig. 7: Inferno X, vv. 32-53.

Fig. 8: Inferno XII, v. 9I. 

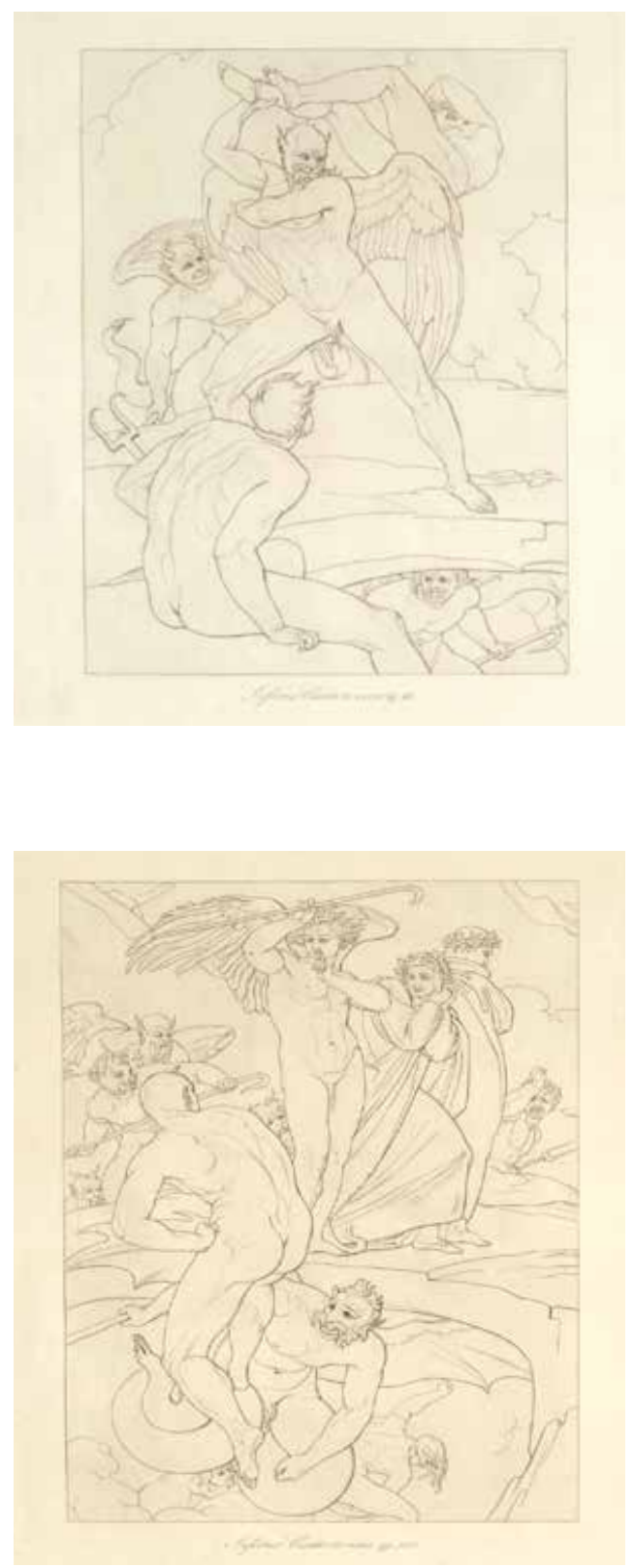

Fig. 9: Inferno XXI, vv. 29-45.

Fig. IO: Inferno XXI, vv. 97-IO5. 

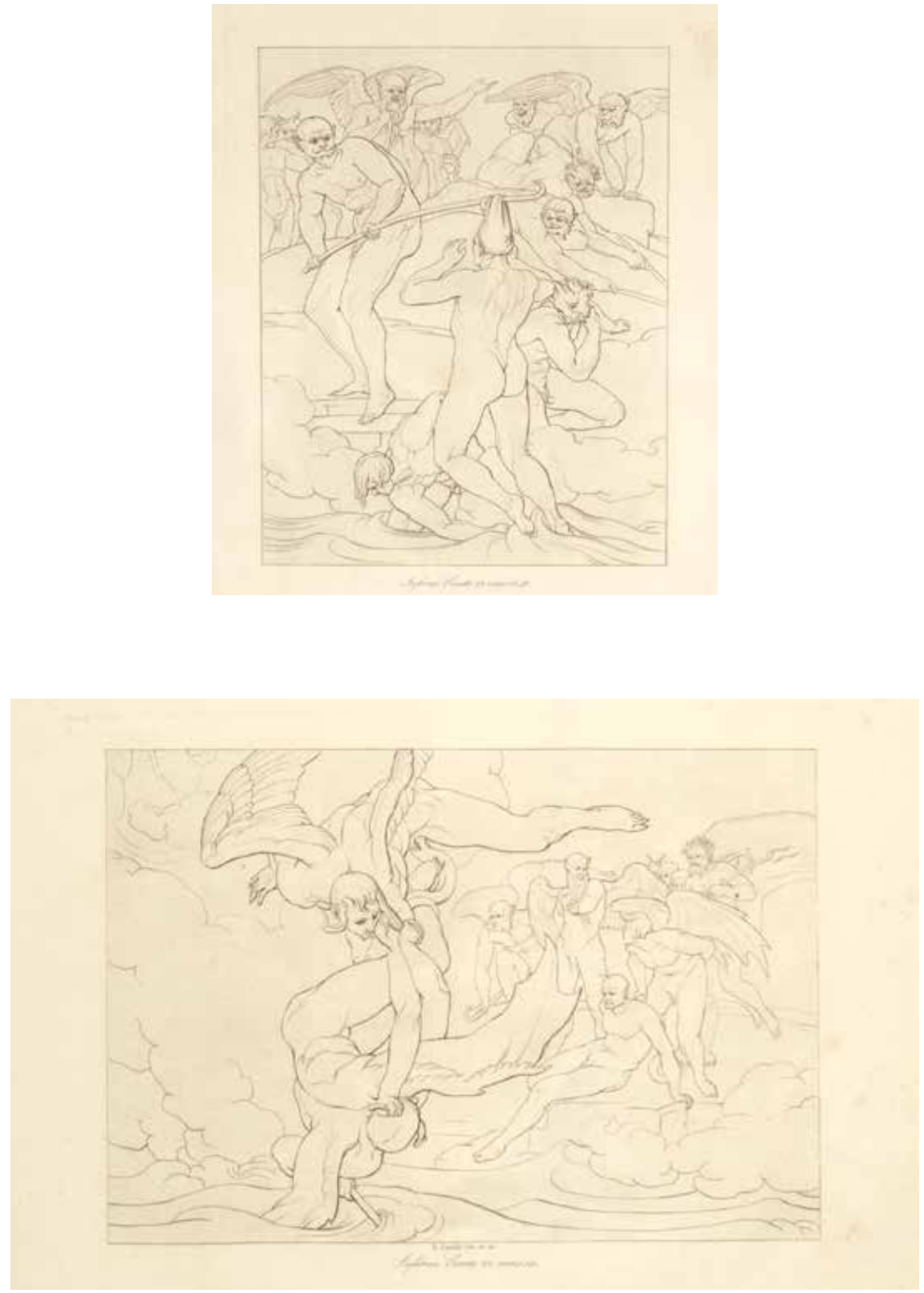

Fig. II: Inferno XXII, vv. 3I-57.

Fig. I2: Inferno XXII, v. I33. 

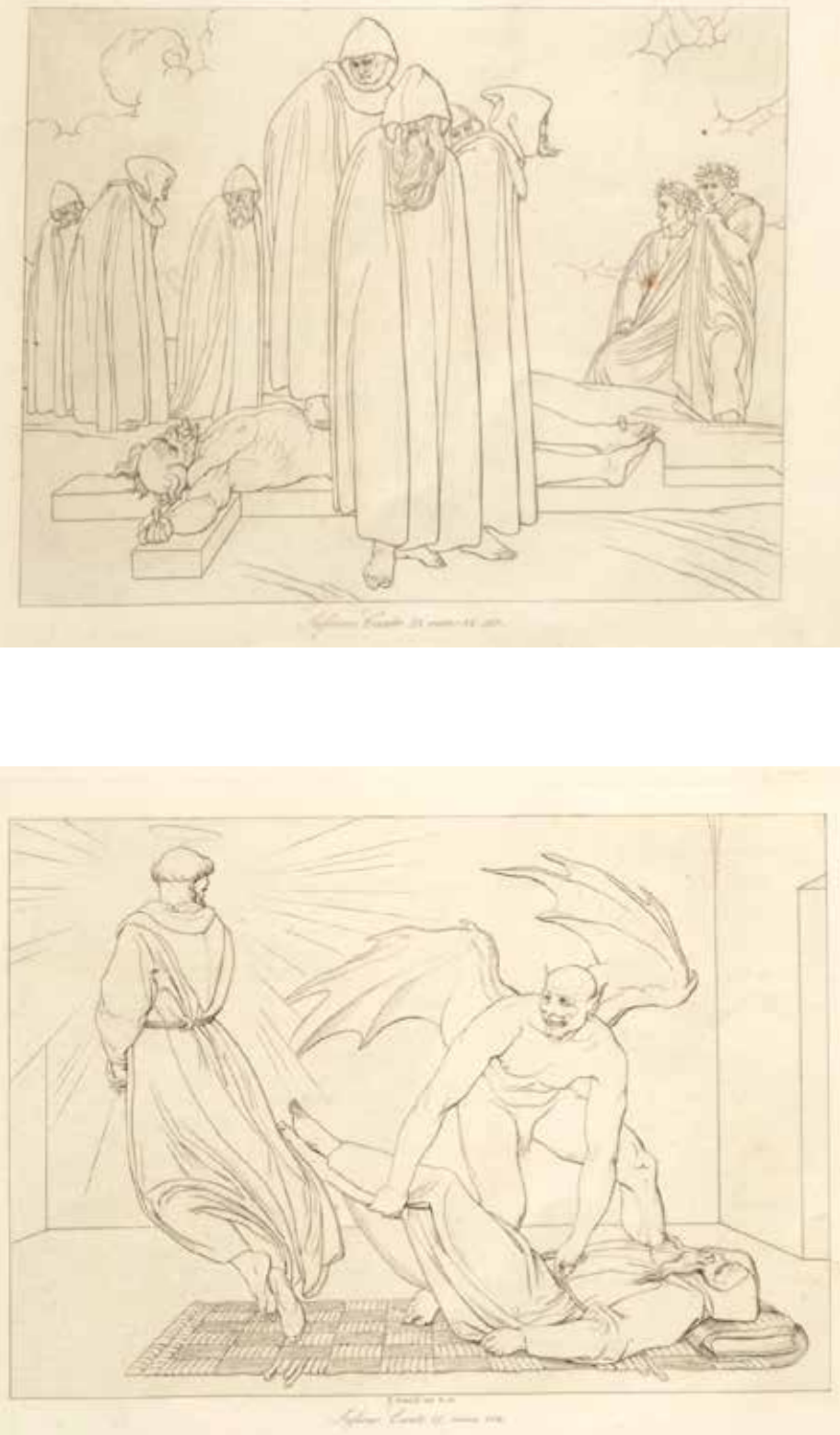

Fig. I3: Inferno XXIII, vv. 58-I20.

Fig. I4: Inferno XXVII, v. II2. 

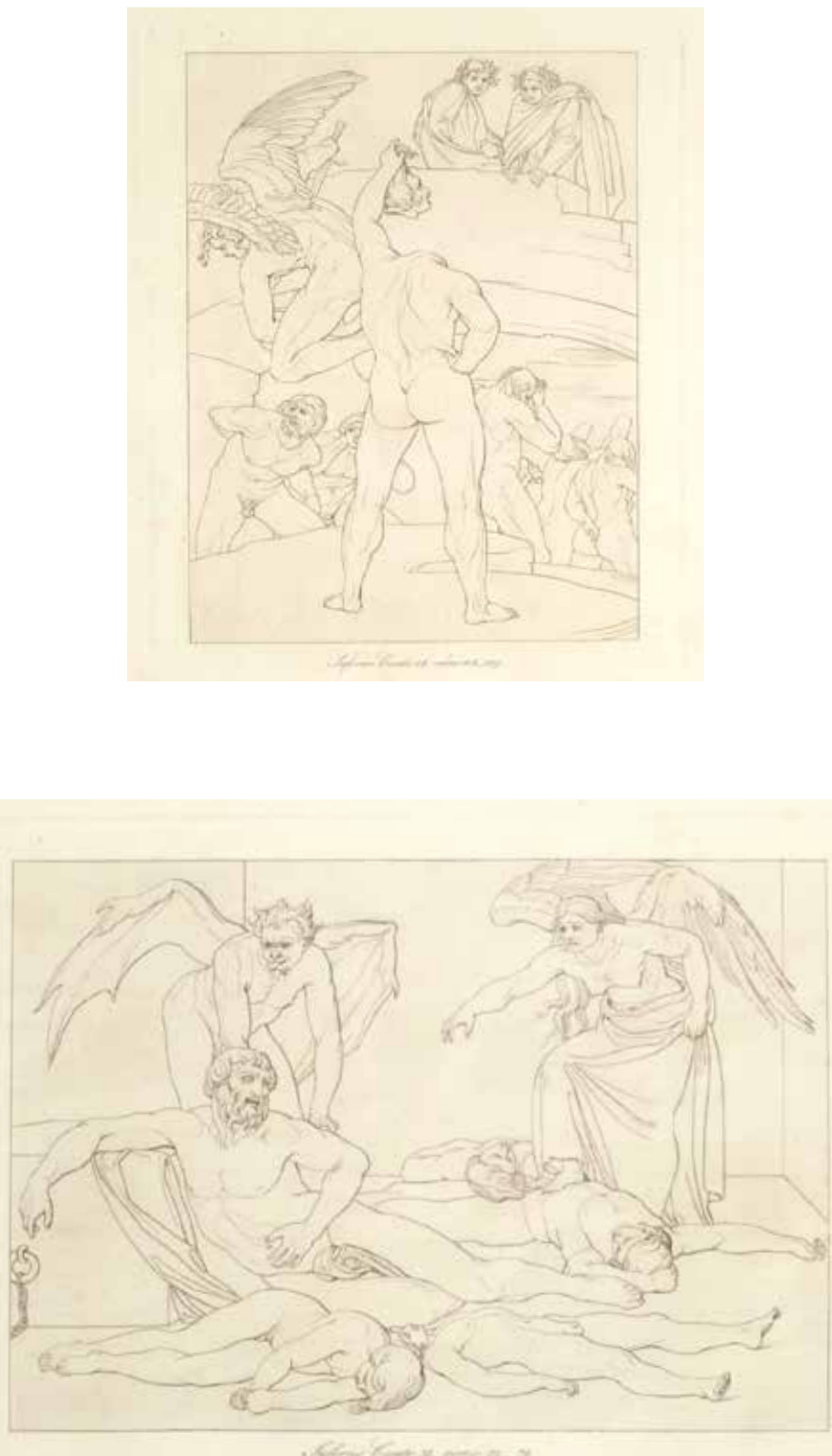

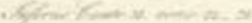

Fig. I5: Inferno XXVIII, vv. II8-I29.

Fig. I6: Inferno XXXIII, vv. 22-75. 


\section{BibLIOGRAFIA}

Crass, H.-M., I98I, Bonaventura Genelli als Illustrator, Bouvier, Bonn.

Cornelius, P., I869, "Bonaventura Genelli. Eine Charakteristik", in Unsere Zeit. Deutsche Revue der Gegenwart, 5, 80I-8I5.

Ebert, H., I96I-62, "Über Buonaventura Genellis Umrisszeichnungen zu Dantes Göttlicher Komödie im Dresdener Kupferstichkabinett, Jahrbuch der Staatlichen Kunstsammlungen, Dresden, 9I-II4.

Ebert, H., I97I, Buonaventura Genelli. Leben und Werk, Weimar.

Hempel, E., I954, "Bonaventura Genellis Umrisse zu Dantes Göttlicher Komödie”, in Deutsches Dante-Jarbuch, XXXIII, 62-86.

Mungen, A., 2006, BilderMusik. Panoramen, Tableaux vivants und Lichtbilder als multimediale Darstellungsformen in Theater-und Musikaufführungen vom Ig. bis zum frühem 20. Jahrhundert, Gardez!, Remscheid.

Nielsen, 2005, E., Bonaventura Genelli. Werk und Kunstauffassung: Ein Beitrag zur Kunst des späten Klassizismus in Deutschland, 2 voll.

Soennecken, I., 2002, Dantes Paolo und Francesca in der Kunst des 19. und 20. Jahrhunderts: Entstehung und Entwicklung eines "romantischen" Bildthemas, Weimar, VDG. 
